\title{
D-MHR: A Distributed Management Scheme for Hybrid Networks to Provide Real-time Industrial Wireless Automation
}

\author{
Pouria Zand, Kallol Das, Emi Mathews and Paul Havinga \\ Pervasive Systems Group, Faculty of EEMCS \\ University of Twente, Enschede, the Netherlands \\ Emails: (p.zand, k.das, e.mathews, p.j.m.havinga)@utwente.nl
}

\begin{abstract}
Current wireless technologies for industrial application, such as WirelessHART and ISA100.11a, use a centralized management approach which makes it difficult and costly for harvester-powered $I / O$ devices to re-join the network in case of power failure. The communication overhead and delay to cope with the dynamic environment of a large-scale industrial network are also very high for an I/O device. In this paper, we therefore propose a distributed management scheme named $D$ MHR, which can address the requirements of energy constrained I/O devices. In D-MHR, the routers can dynamically reserve communication resources and manage the $\mathrm{I} / \mathrm{O}$ devices in the local star sub-networks. We demonstrate that D-MHR achieves higher network management efficiency compared to IS100.11a standard, without compromising the latency and reliability requirements of industrial wireless networks.
\end{abstract}

Keywords-ISA100.11a; IEEE 802.15.4e; Energy harvesting; Distributed management; Hybrid network topology; Resource reservation; Real-time; Process control.

\section{INTRODUCTION}

Input/output (I/O) devices designed for wireless monitoring and process control applications are expected to operate maintenance free for a long time. To enable such working conditions, harvester-powered I/O devices with or without additional power sources are becoming popular. However, current energy harvesters can generate only enough power to allow a limited number of message transmissions/receptions per reporting cycle. Moreover, the availability of the harvested energy often varies in a non-deterministic manner over time. As a result, the harvester-powered I/O devices might shut down unpredictably to recharge and might frequently loose their connection with the network [1].

Several wireless networking standards based on IEEE 802.15.4, such as ZigBee Pro, WirelessHART and ISA100.11a, are developed to support industrial applications. In industrial scenarios, three types of network topologies are commonly considered, namely the star, mesh, and hybrid star-mesh topology. ZigBee Pro is not designed to support industrial process control applications, which have strict latency and reliability requirements [2]. WirelessHART and ISA100.11a are the two standards most widely accepted by the industry. WirelessHART supports full mesh topologies, where all nodes (routers and I/O devices) are considered to have routing capabilities. However, harvester-powered I/O devices are not suitable for routing tasks due to their limited energy budgets. On the other hand, in the ISA100.11a network, I/O devices can be defined as nodes with or without routing capabilities, which results in a hybrid star-mesh topology. This topology is more appropriate for applications with constraint resources. Consequently, we use ISA100.11a as our reference point.

The ISA100.11a standard uses a centralized network management approach, which has several limitations in supporting harvester-powered I/O devices. First of all, an I/O device needs to exchange many messages to re-join, upon loosing its connectivity from the network, which incurs high latency [3]. Secondly, in a harsh and dynamic industrial environment, the quality of links between an I/O device and the router may vary over time. To fix poor/broken links, the central network manager needs to send new instructions over several hops to the network devices, which takes a long time [3]. This problem is further exacerbated as the network scales up.

A distributed network management approach can address the above mentioned challenges in a real-time manner with low overhead. Several studies, such as MC-LMAC [4] and our previous work D-MSR [3], have recently been conducted to address the distributed management approach for industrial systems. However, all of these studies consider the full mesh topology for their proposals, which renders them unfit for hybrid networks. In this paper, we therefore present a Distributed Management scheme for Hybrid networks to provide Real-time industrial wireless automation (D-MHR).

The remainder of this paper is organized as follows. Section II describes the basic concepts and working principles of DMHR. Section III compares performance evaluation metrics of D-MHR with ISA100.11a. Finally, Section IV concludes our work.

\section{D-MHR: NOVEL CONCEPTS AND ARCHITECTURE}

D-MHR supports the hybrid network topology as shown in Figure 1 (a). The network topology has two levels in D-MHR, where the routers form a mesh network, while the I/O devices are part of the local star networks. The radio frequency space is modeled as a matrix of time and channel offset as in IEEE 802.15.4e (TSCH mode) [5]. Time is divided into discrete slots and a collection of time slots creates a superframe. A sample superframe is illustrated in Figure 1 (c).

The routers divide the communication resources among themselves in a distributed manner by listening to the advertisements of their neighbors. Every router includes its own, as well as the communication resources used by its neighbors, in the advertisements. That way, the listeners can select unused resources in their two-hop neighborhood. Routers reserve their resources to enable future communication with $\mathrm{I} / \mathrm{O}$ devices and neighboring routers. The communication resources are defined as a group of consecutive slots in the superframe, referred to as segment. A segment may contain, 1, 2, 4 or 


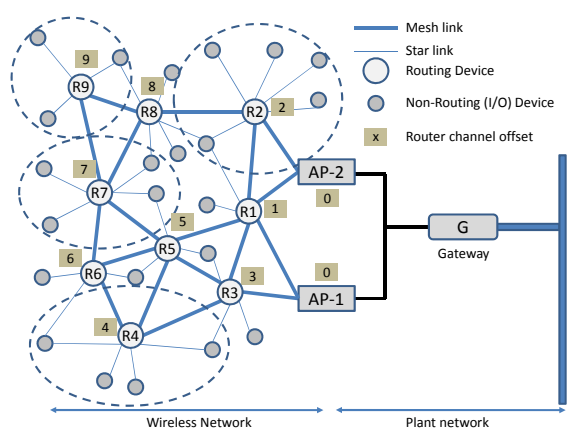

(a)
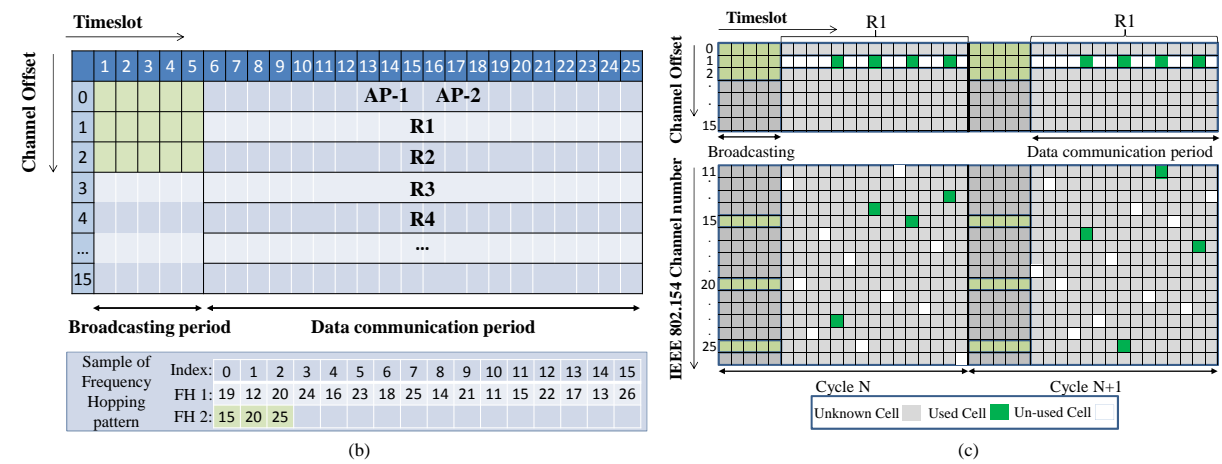

(c)

Fig. 1. (a) D-MHR topology, (b) a sample D-MHR superframe and (c) two cycles of the sample superframe.

any factor of superframe length of slots. A particular router can reserve multiple segments. As the segmentation size decreases, the resource reservation becomes more dynamic and flexible and can support different traffic characteristics of the network. However, small segmentation size increases the management overhead to initiate and update the resource reservation in a distributed manner. Selecting the optimal segmentation size is beyond the scope of this paper and in this work we consider a complete row in the data communication period of the TSCH superframe (i.e., a channel offset) as a segment. Hence, routers divide the communication resources among themselves in this scheme, by selecting different channel offsets as shown in Figure 1 (b). For example, router R1 selects channel offset 1 by following the frequency-hopping pattern illustrated in Figure 1 (b). Router R1 uses physical channel 12 in the first slot of the data communication period, based on the IEEE 802.15.4e physical channel calculation scheme $(\mathrm{FH}[1]=12)$ [5]. Any neighbor, whether an $\mathrm{I} / \mathrm{O}$ device or a router, that wishes to transmit to a router in the same slot, sets its channel to the receiving channel of that router, i.e channel 12 in the example.

The I/O devices in D-MHR first get synchronized with the system and then select the best two routers to provide reliable/redundant paths. The I/O devices use the local statistics of the neighboring routers (e.g. RSSI), as well as the advertised global rank (the qualifying numbers defining the router's individual position relative to other routers with respect to the Gateway) of the routers to choose the best possible routers according to their requirements. To further communicate with the selected routers, the $\mathrm{I} / \mathrm{O}$ devices use the communication resources (segments) reserved by the routers. In order to provide end-to-end real-time communication and to reserve the communication resources toward the final destination, the $\mathrm{I} / \mathrm{O}$ device informs the routers of their traffic characteristics. This includes specified bandwidth and latency information as well as the communication type (periodic or non-periodic). The D-SAR signaling protocol [6] is applied to reserve the required resources along the multipath routes toward the final destination.

Due to channel hopping and multichannel communication, the process of joining and neighbor discovery are challenging issues. Another issue is the scheduling of broadcasting links in a distributed manner. To address these, we modified the TSCH matrix by dividing the superframe into two periods: (i) the broadcasting/advertisement period and (ii) the data communication period as shown in Figure 1 (b). The broadcasting period facilitates neighbor discovery. In the broadcasting period, nodes either broadcast their control messages (e.g. advertisements, routing layer messages) or listen to their neighbor's control messages. As no further unicast communications are scheduled in this period, effective data sharing between the nodes is guaranteed. To facilitate faster neighbor discovery and data sharing during a joining phase (especially for harvester powered I/O devices), we limit the number of channels used in that period to three channels, namely 15,20 , and 25 . These three channels do not overlap with any of the common IEEE 802.11 channels and hence less interference occurs in these channels. In the data communication period, the routers choose particular channel offsets to provide unicast communications. The network devices may in turn use the broadcasting and data communication periods to create a superframe of any length that is an even multiple of a basic superframe length (e.g. 250 $\mathrm{ms})$, in which these periods are repeated.

\section{A. D-MHR protocol stack architecture}

The protocol stacks of ISA100.11a and D-MHR are shown in Figure 2. In ISA100.11a, a central system manager schedules all communications and constructs all routes through the data link layer management object (DLMO). It also establishes end-to-end connections in the network through the transport layer management object (TLMO). In contrast, the network setup is performed in a distributed manner in D-MHR.

The new sub-layers, modules and tables of our proposed D-MHR protocol stack are highlighted in Figure 2 (b). The

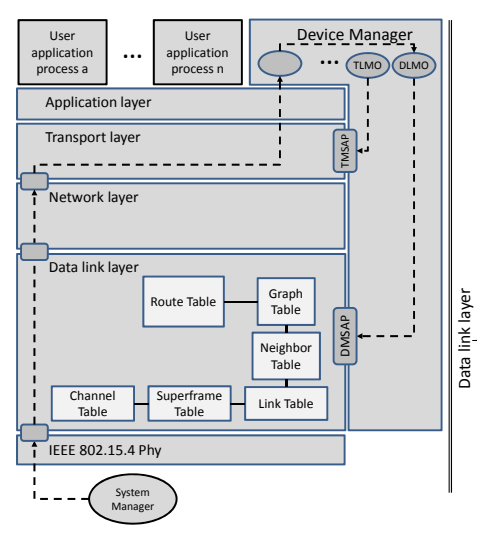

(a)

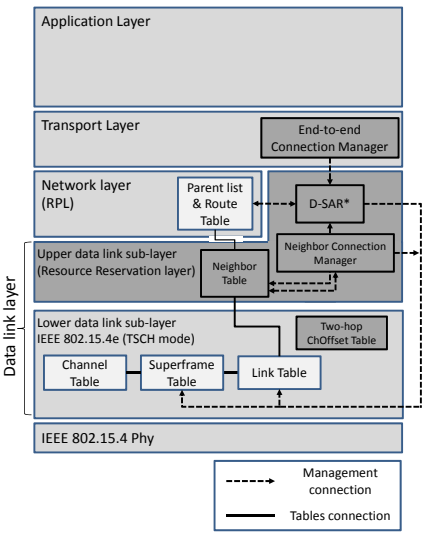

(b)
Fig. 2. The protocol stacks of (a) ISA100.11a and (b) D-MHR. 


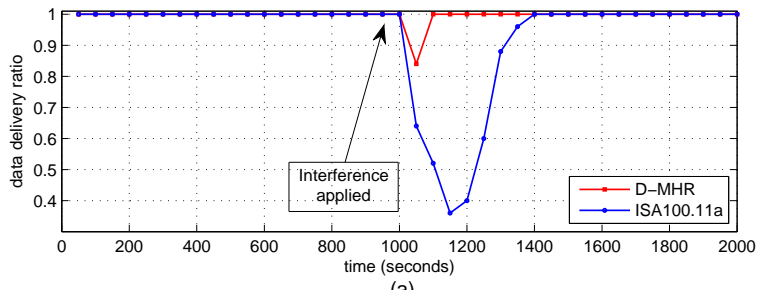

(a)

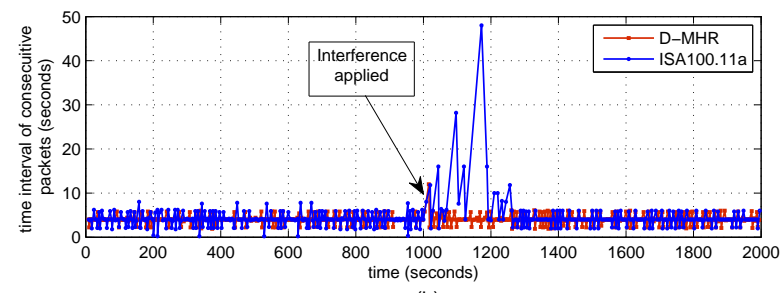

(b)

Fig. 3. Reliability and real-time guarantee.

data link layer consists of two sub-layers: the lower and the upper data link sub-layer. In the lower data link sub-layer, we modify the IEEE $802.15 .4 \mathrm{e}$ (TSCH mode) standard to fit our requirements. A Two-hop Channel Offset table is added in this layer enable the allocation of the communication resources to the routers and to enable the scheduling of interference-free communications in the network. In the upper data link sublayer (the resource reservation layer), we implement D-SAR signaling protocol that is designed to reserve the resources in the multi-path routers. We also implement the neighbor connection manager modules, that is designed to define the initial communication link between the network devices [3]. This helps to configure the communication tables locally in the lower data link sub-layer. Additionally, the Neighbor table containing neighbor statistics, is implemented in this sub-layer. In the network layer, Routing Protocol for Low power and Lossy Networks (RPL) is used with proper adjustments [7]. The End-to-end Connection Manager module is implemented in the transport layer. This module establishes the end-to-end connection through the D-SAR signaling protocol.

\section{PERFORMANCE EVALUATION}

\section{A. Simulation setup}

Both the D-MHR and ISA100.11a protocol stacks are implemented in NS-2. We consider a network of $38 \mathrm{I} / \mathrm{O}$ devices, 22 routers, 2 access points and 1 gateway in an area of $80 \times 40 \mathrm{~m}^{2}$. The routers are systematically placed in the network, whereas the I/O devices are randomly distributed. The transmission range of all nodes are set to $15 \mathrm{~m}$. We use the two-ray ground radio model in the simulation. The constant bit rate (CBR) traffic model is employed to generate the sensor data in our simulation.

\section{B. Reliability and real-time guarantee}

To evaluate the reliability and real-time guarantee of DMHR and ISA100.11a in the presence of external interference, we introduce failures between $\mathrm{I} / \mathrm{O}$ devices and routers in the star sub-network. After this, the packet delivery ratio and the time interval of the consecutive packets are calculated at the destination. Figure 3 (a) illustrates that the packet delivery ratio suddenly drops for both approaches when we apply the external interference. However, compared to D-MHR, it takes longer for the ISA100.11a standard to reach back to the stable state. Figure 3 (b) shows the jitter in the time interval of the consecutive packets received at the final destination. It varies slightly around the expected value of four seconds (data publishing interval) in normal operations. When the interference is applied, the jitter in ISA100.11a dramatically increases and requires considerably more time to reach back to the normal values than in D-MHR. In ISA100.11a, the system manager has to perform repairs on receiving the periodic neighbor diagnostic reports, which takes time. On the other hand, in D-MHR, the I/O devices can use their local statistics to fix the problem, which improves the reliability and real-time aspects of our approach.

\section{CONCLUSION}

This paper presented a distributed network management scheme for hybrid networks named D-MHRs, which can support industrial applications by providing reliable and real-time communication. D-MHR can achieve a lower latency in data delivery than ISA100.11a. Moreover, D-MHR can fix the network problem more quickly and with less message exchanges overhead in case of internal and external interference than the ISA100.11a standard. Thus, D-MSR can better support the monitoring and process control applications in industrial automation, including energy constrained I/O devices (e.g., harvester powered ones). Our future work will focus on the further evaluation of D-MHR and ISA100.11a in real industrial scenarios.

\section{ACKNOWLEDGMENTS}

This research is supported, in part, by the EU FP7-ICT project WiBRATE (http://wibrate.eu), under the Grant No. 289041.

\section{REFERENCES}

[1] Z. A. Eu, H.-P. Tan, and W. K. G. Seah, "Opportunistic routing in wireless sensor networks powered by ambient energy harvesting," Comput. Netw., vol. 54, no. 17, pp. 2943-2966, Dec. 2010. [Online]. Available: http://dx.doi.org/10.1016/j.comnet.2010.05.012

[2] P. Zand, S. Chatterjea, K. Das, and P. Havinga, "Wireless industrial monitoring and control networks: The journey so far and the road ahead," Journal of Sensor and Actuator Networks, vol. 1, no. 2, pp. 123-152, 2012. [Online]. Available: http://www.mdpi.com/2224-2708/1/2/123

[3] P. Zand, A. Dilo, and P. Havinga, "D-MSR: A distributed network management scheme for real-time monitoring and process control applications in wireless industrial automation," Sensors, vol. 13, no. 7, pp. 8239-8284, 2013. [Online]. Available: http://www.mdpi.com/1424$8220 / 13 / 7 / 8239$

[4] O. D. Incel, L. van Hoesel, P. Jansen, and P. Havinga, "MC-LMAC: A multi-channel MAC protocol for wireless sensor networks," Ad Hoc Netw., vol. 9, no. 1, pp. 73-94, Jan. 2011. [Online]. Available: http://dx.doi.org/10.1016/j.adhoc.2010.05.003

[5] IEEE Standard for Local and metropolitan area networks-Part 15.4: Low-Rate Wireless Personal Area Networks (LR-WPANs) Amendment 1: MAC sublayer, Std., 2012.

[6] P. Zand, S. Chatterjea, J. Ketema, and P. Havinga, "A distributed scheduling algorithm for real-time (D-SAR) industrial wireless sensor and actuator networks," in IEEE 17th Conference on Emerging Technologies Factory Automation (ETFA), 2012, pp. 1-4.

[7] T. Winter, P. Thubert, A. Brandt, J. Hui, R. Kelsey, P. Levis, K. Pister, R. Struik, J. Vasseur, and R. Alexander, RPL: IPv6 Routing Protocol for Low-Power and Lossy Networks, Internet Engineering Task Force (IETF) Std. 2070-1721, Mar. 2012. 